\title{
The GVS: Glasgow Coma Score, Volume \& Site of Hemorrhage as a Simple Scoring System for Intracerebral Hemorrhage
}

\author{
Sudipta Kumer Mukherjee ${ }^{1 *}$, Ruhul Quddus ${ }^{2}$, Mohsin Ali Farazi ${ }^{3}$, Mohammad Nazrul Islam ${ }^{4}$ \\ ${ }^{1}$ Assistant Professor, Department of Pediatric Neurosurgery, National Institute of Neurosciences \& Hospital, Dhaka \\ ${ }^{2}$ Assistant professor, Department of Neurology, Shahid Sheikh Abu Naser specialized Hospital, Khulna \\ ${ }^{3}$ Assistant Professor,Department of Neurosurgery, Shahid Sheikh Abu Naser Specialized Hospital, Khulna \\ ${ }^{4}$ Assistant Professor, Department of Radiology and Imaging, National Institute of Neurosciences \& Hospital, Dhaka
}

Received: June 10, 2016; Accepted: July 26, 2016; Published: August 26, 2016

*Corresponding author: Sudipta Kumer Mukherjee, Assistant professor, Pediatric Neurosurgery, National Institute of neurosciences \& Hospital (NINS\&H), Dhaka, Bangladesh, Tel: +8801711-709096; E mail: neudipta@gmail.com

\begin{abstract}
Background and purpose: Hemorrhage within the brain parenchyma (ICH) has the worst impact on human life and accounts for $10-17 \%$ of all strokes seen in the mid and later age of life. The goals of this research were to develop a new score to predict mortality and morbidity of ICH also to check the efficacy and simplicity of the new score.
\end{abstract}

Methods: We performed a prospective observational cohort study of all patients with spontaneous ICH admitted to the inpatient departments of KMCH, KSH and IBH from June 1, 2010, to May 31, 2011 and follow-up for each patient was for next one year where patient assessed with the modified Rankin Scale (mRS) at 1 month, 3 months, and one year. The proposed score (GVS) was developed by check the association of independent variable and outcome. We also checked the efficacy of proposed scoring system; compare this one with previous conventional scoring system.

Results: Of 209 total patients, 56 were lost to follow-up, thus we concluded the study with 153 cases. GVS was significant at $\mathrm{p}=$ $<0.05(95 \% \mathrm{CI})$, CAT-PCA test revealed age, midline shift, and IVH weakly related with outcome. The interrater reliability for the raters was found to be Kappa $=0.75(p<0.001)$ reveal substantial agreement between GVS and ICH. Change in outcome after three months is less though there were some noticeable changes from three months to one year.

Conclusions: The GVS is a simple scoring system for outcome prediction after spontaneous ICH.

Keywords: Intracerebral hemorrhage; Score; Outcome; GCS

\author{
Abbreviations \\ AVM- Arterio-Venous Malformation. CATPCA- Categorical \\ Principal Components Analysis. DM- Diabetes Mellitus. ED- \\ Emergency Department. FUNC- Functional. GCS- Glasgow Coma \\ Score. HTN- Hypertension. IBH- Islami Bank Hospital . ICH- \\ Intracerebral Hemorrhage. IVH- Intraventricular Hemorrhage.
}

KMCH- Khulna Medical College Hospital. KSH- Khulna Surgical Hospital. MRS- Modified Rankin Score.

\section{Introduction}

Spontaneous ICH, which accounts for $10-15 \%$ of all cases of stroke, is associated with high rate of morbidity, and fewer than half of the affected patients surviving the first year [1]. In the days and weeks following bleeding, the patient's clinical course largely depends on several factors. The level of consciousness, the size of the clot, the presence and degree of shift and evidence of ventricular rupture are the most important prognostic indicators [2]. While ICH may represent a low percentage of the overall number of strokes, ICH carries a 30 day mortality of $35 \%$ to $52 \%$ making itself a serious concern for emergency physician, neurologist, neurosurgeon and neurocritical care specialist throughout the world [3]. Important prognostic factors especially for mortality rate vary widely and depend on size and location of clot, age and medical condition of patient, and etiology of the hemorrhage. Systemic disorders such as HTN, DM or specific organ dysfunction have an indirect significant impact on survival during sub acute period [2]. Our current study focuses on clinical and radiological parameters of patients on presentation and their recovery.

\section{Methodology}

209 patients from June 1, 2010 to May 31, 2011 at KMCH, IBH and KSH in Khulna, Bangladesh, were enrolled for this study. Patients were identified in the ED and during in-hospital care. All patients were treated with a standard stroke care guideline. Informed consent was obtained from all patients or their legal representatives. Permission was obtained from either ethical committee or the head of the institution. Data were collected by the authors. Data included Age, GCS, hematoma volume, presence or absence of IVH and location of hematoma. 
The proposed scoring system is entitled as "GVS" (Glasgow Coma Score, Volume and Site of hematoma) and is the sum of individual points described as follows: GCS- $(3-4=2,5-12=1$, $13-15=0)$, Volume $-(>30 \mathrm{cc}=1,<30 \mathrm{cc}=0)$, Site $-($ Brain stem $=2$, $\mathrm{IVH}=1$, cerebellar $=1$, supra tentorial $=0$ ), Patient GVS Scores for $\mathrm{ICH}$ ranged from 0 to 5 . Whereas Hemphill intracerebral hemorrhage scores (HICH) scores ranged from 0 to 6 [4]. The formula for standard volume measurement of an ellipsoid was used to compute volumes of hematoma [5]. Both clinical care team and radiologist simultaneously collected the data. Outcome was assessed by MRS during follow- up visits during one month, three months and one year after onset of symptom by direct contact with the patient or patient's family or local physicians. The patients lost to follow-up during 12-months were excluded from study. Midline shift, age and co-morbidity were analyzed independently with MRS. their correlation was very weak in this study. That is why this parameter was not included in the GVS scoring system. Too many outcome prediction key in the MRS made the calculation complicated, so the outcome rearranged as good outcome (MRS 0,1,2), moderate outcome (MRS 3, 4, 5) and Death (MRS 6) for this study

\section{Statistics}

All data were analyzed using SPSS: 15. A statistically significant difference was indicated by a probability value $\mathrm{p} \leq$ 0.05 (95\% confidence interval). Chi-square test was carried out between each score and outcome for both systems. The association between outcome and different variable was analyzed by CATPCA. The relationship between both scores was determined by the kappa test.

Rationale of the study: Since ICH presents with various clinical features, e.g. site, size and outcome among the patients, we cannot predict to patients, their relatives and hospital administrators regarding the patient's outcome. Multiple scoring systems predicting the outcome of patients with ICH have been identified; the most widely accepted are the Hemphill ICH score and the FUNC score $[4,6]$. The conventional scoring system involved complex memorization and calculation especially in ED. This research work was undertaken to develop and validate a new outcome prediction score which is simply calculated and expressed at the bedside.

Objectives: The goal of the study was to develop a simple and effective scoring system for ICH outcome regarding mortality and morbidity prediction.

PatientSelection: Patients with spontaneous ICH irrespective of age and sex were enrolled in this study. Patients with ICH secondary to tumors, trauma, and hemorrhagic transformation of cerebral infarction, ruptures of aneurysm or AVM were excluded. Patients who refused to participate in this research and who were evaluated 24 or more hours after onset of symptoms were also excluded from the same study. As per these inclusion and exclusion criteria the sample size (n) was 209 patients.

\section{Results}

Initially data were recorded from 209 cases by selection criteria, over one year $26.7 \%(n=56)$ patients were lost to follow up. Thus we concluded our study with 153 patients.

\section{Discussion}

Prognostic indicators have an important role in clinical judgment. It is important to identify patients with very poor prognosis who are unlikely to benefit from treatment: patients who would otherwise have died may benefit only by surviving, but may be completely dependent and have a poor quality of life. Ideally, any method of predicting outcome should be reliable, simple, accurate and reproducible [7]. However, several models for use in the case of spontaneous ICH are complex algebraic equations for outcome prediction, and none have been simplified into a standard clinical grading scale analogous to the GCS, Hunt-Hess, or Spetzler-Martin scales. It is likely that this lack of a uniform ICH scale has contributed to variability in enrollment criteria for ICH studies as well as to heterogeneity in clinical ICH care. In essentially every clinical grading scale there exists a compromise between simplicity and accuracy of outcome prediction. To strike the appropriate balance between these two factors, the general purpose of the grading scale must be considered. The GVS is a clinical grading scale composed of factors related to a basic neurological examination (GCS) and initial neuroimaging (ICH volume, IVH, site). The purpose of this grading scale is to provide a standard assessment tool that can be easily and rapidly determined at the time of ICH presentation by physicians without special training in stroke neurology $[4,8]$. The use of the conventional ICH score is not easy especially in the ED because the many variables make for a complex calculation. The purpose of this study was to develop a new ICH outcome prediction model and compare simplicity and accuracy with previous prediction models. Three letters GVS (G = Glasgow Coma Score, $\mathrm{V}=$ Volume of blood, $\mathrm{S}=$ Site of hematoma) coined from first capital letter of its component words, seem easy to memorize. The small number of variables makes it is easy to calculate the GVS, especially in the ED.

The GVS was significant $\mathrm{p}<0.05(95 \% \mathrm{CI})$ in all three time points (one month, three months and one year) in this study. Therefore the GVS is an effective clinical grading scale for stratifying likelihood of favorable functional outcome throughout the first year after spontaneous ICH.

The CATPCA test was done to check the strength of association between the different variables and outcome, and revealed that age, midline shift and IVH are weakly related to outcome.

The GCS score is now a standard neurological assessment tool that is reproducible and reliable. ${ }^{10}$. Because the GCS score is overwhelmingly the strongest outcome predictor in acute ICH, weighting this component of the ICH Score more than others is justified, and dividing it into these three groups is more clinically meaningful than dichotomizing toward the middle of the range of possible GCS scores (range 3 to 15). [4]

Age has been found to be an independent predictor of ICH outcome in some prior prediction models, while age has not 
been associated with outcome in others [4]. In the present series statistical analysis revealed age to be weakly related with outcome ( $p=0.598$ at 1 month, $p=0.817$ at 3 months and $\mathrm{p}=0.902$ at 1 year). Thus we exclude age from GVS scoring system. The fact that age has been an inconsistent ICH outcome predictor among various models and may have its strongest influence among the group of very elderly patients suggests two possibilities. Either the very elderly sustain worse neurological injury from ICH irrespective of size or location, or overall medical care decisions in elderly patients are less aggressive even if ICHrelated neurological injury is not as profound. [4]

ICH volume is consistently associated with outcome in ICH prediction models $[8,11]$. Often ICH volume has been divided into three groups representing small, medium, and large hematoma size $[8,11]$. While the specific volume cut points vary depending on the specific model, small hematomas haven often been considered as $<30 \mathrm{~cm}^{3}$ and large hematomas as $>60$ $\mathrm{cm}^{3}[8,12]$. While ICH volume is a component of the ICH Score, its association with outcome was not as strong as some other predictors. In fact, ICH volume was not an independent predictor for outcome in infratentorial hemorrhages. This may be because small hemorrhages in the brain stem or cerebellum may have catastrophic consequences, making location, not size, and the more important predictor for infratentorial ICH. Additionally, while larger supratentorial ICH volumes were associated with increased mortality, the addition of a "large hematoma" group did not improve the model because patients with larger hematomas who died also had other predictors such as low GCS score, advanced age, or IVH that influenced outcome to a greater degree. This has practical implications for patient treatment in that we believe that the logistic regression model and ICH score derived from this cohort would not justify exclusion of a patient for treatment solely on the basis of a large hematoma in the absence of other poor outcome predictors such as low GCS score, advanced age, or IVH. Thus, the ICH volume component of the ICH score is dichotomized to reflect the strength of association with outcome and weighted accordingly. [4]

The presence of any IVH and infratentorial hemorrhage origin were the other factors independently associated with 30-day mortality in this cohort therefore included in the ICH score. Both are easy to assess and are dichotomous variables. Undoubtedly, further characterization of the degree of IVH and IVH-associated hydrocephalus could provide additional prognostic information, [13] but these are also more subjective measures that are more complicated to assess and therefore were not included in this model.

We believed that it was important to create a single model that would include all ICH and not limit the assessment to supratentorial ICH, as in some other models $[11,12,15,16]$. Including a term for infratentorial hemorrhage and selecting the cut point for ICH volume as previously described allowed this to be accomplished. Cross tabulation between Site and ICH revealed Site $(p=0.069$ at 1 month, $p=0.141$ at 3 months, and $p=0.321$ at 1 year). Other factors may have prognostic value after ICH, such as medical co-morbidities and midline shift. These were not included in the ICH Score because they are not strongly related with outcome.

This study also helps to elucidate the course of recovery, which is important clinically because it helps the caregiver to make a plan for ongoing care needs. The pace of recovery during the first year after ICH improvement takes several forms. First, many patients show profound improvement, often from very severe disability, during the first one month. However, improvement also occurs in many patients across the disability spectrum up to the first three months. Additionally this study demonstrates that a substantial proportion of patients with spontaneous ICH improved after three months and that this improvement occurs throughout the first year in patients who were less disabled.

In general, an increase in both these scores (GVS \& HICH) was associated with a lower likelihood of favorable outcome. The inter-rater reliability for both the GVS and the HICH was found to be Kappa $=0.75(\mathrm{p}<0.001)$. That indicates statistically both the GVS and the HICH carry similar importance.

In a published series by Hemphill reporting the outcome of patients with ICH, on presentation age was found to be a particularly important prognostic factor.[4] However, we assessed all recorded variables with outcome by CATPCA, and found that age was very weakly related with outcome. Thus we excluded age from the scoring system. Several prognostic models for ICH have been previously developed and validated [12-14]. These models have found several characteristics associated with outcome, as measured by mortality and functional outcome. Among these various characteristics, level of consciousness on hospital admission (often assessed as GCS score) and hematoma volume have usually been the most robust outcome predictors, with other factors, such as presence and amount of IVH, also associated with outcome in some models [11, 12, 15]. The GVS and HICH Scores accurately stratified patients at each of these cut points ( $\mathrm{p}<0.05$, test for trend).

This study has certain limitations, e.g. lack of controls in the treatment protocol and pre-ICH cognitive impairment was not included. To establish a clinically applicable score system, we want to overcome these limitations by following a fixed treatment protocol, including pre ICH cognitive status in a randomized multicenter study. Important questions have been raised for the high score group whether these patients should be treated aggressively, and whether they have any chance of a meaningful recovery. We also want to validate this score by future multicenter randomized study. After such a study this GVS score may be useful for outcome prediction for spontaneous ICH in the clinical setting.

\section{Conclusion}

We offer an outcome prediction score for spontaneous ICH patient, which has been titled the GVS. The GVS is a simple and statistically valid scoring system additionally, the name GVS has an inherent opportunity for memorization, because this is an abbreviation of its components. 
Table1: Characteristics of enrolled patients.

\begin{tabular}{|c|c|c|}
\hline \multicolumn{2}{|l|}{ Variable } & Number \\
\hline \multicolumn{2}{|l|}{ Age } & $\begin{array}{l}56 \text { to } 70 \text { age group } 81 \\
(53 \%)\end{array}$ \\
\hline \multirow{3}{*}{ GCS } & 3 to 4 & $23(15 \%)$ \\
\hline & 5 to 12 & $68(44.4 \%)$ \\
\hline & 13 to 15 & $62(41.5 \%)$ \\
\hline \multirow{5}{*}{ Locations } & Supra tentorial & $101(66 \%)$ \\
\hline & Supra and infra tentorial & $4(2.6 \%)$ \\
\hline & Cerebellar & $5(3.3 \%)$ \\
\hline & Brain stem & $13(8.5 \%)$ \\
\hline & Ventricular & $30(19.6 \%)$ \\
\hline \multirow{2}{*}{ Volume } & $>30 \mathrm{cc}$ & $56(36.6 \%)$ \\
\hline & $<30 \mathrm{cc}$ & 97 (63.4\%) \\
\hline \multicolumn{2}{|c|}{ Midline Shift } & $84(54.9 \%)$ \\
\hline \multirow{3}{*}{ H/O HTN } & Present & $112(73.2 \%)$ \\
\hline & Absent & $27(17.6 \%)$ \\
\hline & History not available & $14(9.2 \%)$ \\
\hline \multicolumn{2}{|l|}{ DM } & $38(24.8 \%)$ \\
\hline
\end{tabular}

Among the 153 patients, mean age at ICH $61.25 \pm 2.33$ years, the majority $81 / 153$, (53\%) was in the 56 to 70 years age group. GCS score, ICH volume, midline shift and sites on initial CT scan described in Table 1.

Table 2: GVS and one month outcome cross tabulation.

\begin{tabular}{|c|c|c|c|c|c|}
\hline & \multicolumn{3}{|c|}{ Outcome (after one month) } & \multirow[b]{2}{*}{ Total } \\
\hline & & \begin{tabular}{|l} 
M6 \\
(Death)
\end{tabular} & $\begin{array}{l}\text { Moderate outcome } \\
(\mathrm{m} 3,4,5)\end{array}$ & $\begin{array}{l}\text { Good outcome } \\
(\mathrm{m} 0,1,2)\end{array}$ & \\
\hline \multirow{5}{*}{ 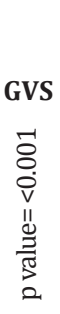 } & GVS 0 & $7(21 \%)$ & $19(56 \%)$ & $8(24 \%)$ & 34 \\
\hline & GVS 1 & $13(32 \%)$ & $23(56 \%)$ & $5(12 \%)$ & 41 \\
\hline & GVS 2 & $27(64 \%)$ & $8(19 \%)$ & $7(16 \%)$ & 42 \\
\hline & GVS 3 & $19(66 \%)$ & $9(30 \%)$ & $1(4 \%)$ & 29 \\
\hline & GVS 4 & $6(86 \%)$ & $1(14 \%)$ & 0 & 7 \\
\hline \multicolumn{2}{|c|}{ Total } & $72(47 \%)$ & $60(39.2)$ & 21(13.7\%) & 153 \\
\hline
\end{tabular}

After one month 72 died; 81 patients survived with 21 in good and 60 in moderate condition. Both GVS and HICH scores showed a p value $<0.001$, i.e. So, both were significant at one month follow-up.

Table 3: GVS and three months outcome cross tabulation.

\begin{tabular}{|c|c|c|c|c|c|}
\hline & \multicolumn{3}{|c|}{ Outcome (after three months) } & \multirow{2}{*}{ Total } \\
\hline & & Death & $\begin{array}{l}\text { Moderate outcome } \\
(\mathrm{m0}, 1,2)\end{array}$ & $\begin{array}{l}\text { Good outcome } \\
(\mathrm{m} 3,4,5)\end{array}$ & \\
\hline \multirow{5}{*}{ 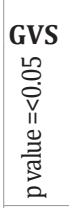 } & GVS 0 & $11(32 \%)$ & $9(26 \%)$ & $14(42 \%)$ & 34 \\
\hline & GVS 1 & $21(52 \%)$ & $10(24 \%)$ & $10(24)$ & 41 \\
\hline & GVS 2 & $30(71 \%)$ & $4(9 \%)$ & $8(20 \%)$ & 42 \\
\hline & GVS 3 & $23(80 \%)$ & $4(13 \%)$ & $2(7 \%)$ & 29 \\
\hline & GVS 4 & $6(86 \%)$ & $1(14 \%)$ & 0 & 7 \\
\hline \multicolumn{2}{|c|}{ Total } & 91 & 28 & 34 & 153 \\
\hline
\end{tabular}

After three months 91 died; 62 patients survived with 34 in good and 28 in moderate condition. GVS showed a p value $=0.003$ and HICH scores showed a $p$ value $<0.001$. i.e. - both were significant $(p<0.05)$ on three months follow-up.
Table 4: GVS and one year outcome cross tabulation.

\begin{tabular}{|c|c|c|c|c|c|}
\hline & \multicolumn{3}{|c|}{ Outcome (after one year) } & \multirow[b]{2}{*}{ Total } \\
\hline & & $\begin{array}{l}\text { Death } \\
\text { (m6) }\end{array}$ & $\begin{array}{l}\text { Moderate outcome } \\
(\mathrm{m} \mathrm{3,4,5)}\end{array}$ & $\begin{array}{l}\text { Good outcome } \\
(\mathrm{m} \mathbf{0 , 1}, 2)\end{array}$ & \\
\hline \multirow{5}{*}{ 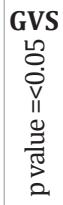 } & GVS 0 & $12(35 \%)$ & $7(21 \%)$ & $15(44 \%)$ & 34 \\
\hline & GVS 1 & $24(59 \%)$ & $6(15 \%)$ & $11(27 \%)$ & 41 \\
\hline & GVS 2 & $30(71 \%)$ & $4(10 \%)$ & $8(19 \%)$ & 42 \\
\hline & GVS 3 & $23(79 \%)$ & $3(10 \%)$ & $3(10 \%)$ & 29 \\
\hline & GVS 4 & $7(100 \%)$ & 0 & 0 & 7 \\
\hline \multicolumn{2}{|c|}{ Total } & $96(62 \%)$ & $16(11 \%)$ & $41(27 \%)$ & 153 \\
\hline
\end{tabular}

After one year 91 patients died; 57 patients survived with 16 in good and 41 in moderate outcome. GVS showed a $p$ value $=0.008$ and $\mathrm{HICH}$ scores showed a $\mathrm{p}$ value $=0.002$. i.e. - both are significant $(\mathrm{p}<0.05)$ on one year follow-up.

Table 5: Outcome summary.

\begin{tabular}{|l|l|l|l|l|}
\hline & Panel & \multicolumn{2}{l|}{ Outcome } & \\
\cline { 3 - 5 } & & Death (m6) & $\begin{array}{l}\text { Moderate } \\
\text { outcome }\end{array}$ & $\begin{array}{l}\text { Good } \\
\text { outcome }\end{array}$ \\
\hline After 1 month & A & $72(47 \%)$ & $60(39 \%)$ & $21(14 \%)$ \\
\hline After 3 months & B & $91(59 \%)$ & $28(18 \%)$ & $34(22 \%)$ \\
\hline After 1 year & C & $96(62 \%)$ & $16(11 \%)$ & $41(27 \%)$ \\
\hline
\end{tabular}

In panel A, 81 (53\%) patients survived for one month after ICH; in panel B, after 3 months patients survival diminished to $62(40 \%)$ which was further diminished to 57 (38\%)showed in panel C. 60 (39\%) had a moderate outcome at one month diminishing to $16(11 \%)$ at one year, so $44(28.7 \%)$ patients either improved or died by that time. Regarding death, $72(47 \%)$ died within one month; at one year that number increased to 96 (62\%), so from one month to one year newly 24/153 (15\%) died. 21/153 (13.7\%) of patients after one month showed good outcome increasing to $41(27 \%)$ after one year, i.e. - 20/153 (13\%) of patients improved between one month and one year. There is a noticeable change between panels A and B but very minor change was noticed between panel B and C. This outcome trend indicates that most of the patients achieved optimum outcome level within three months. Only a very small number of patients changed outcome categories between three months and one year. This is especially notable that patients being severely impaired even after $1^{\text {st }}$ month improved or worsened equally and rest was in static situation.

\section{Acknowledgement}

Professor R. Andrew for checking Neurosurgical details, Mr. Hannan for grammatical checking and Mr. Rajot for assistance in data collection.

\section{References}

1. Nilsson OG, Polito A. Are primary supratentorial intracerebral hemorrhages surrounded by biochemical penumbra? A microdialysis study. Neurosurgery. 2006;59(3):521-528.

2. Kaufman HH. Spontaneous intraparenchymal brain hemorrhage. In: Wilkins RH, Rengachary SS,eds. Neurosurgery. $2^{\text {nd }}$ ed. New York, NY: McGraw-Hill. 1996;2567-2581.

3. Brott T, Broderick J, Kothari R, William Barsan, Thomas Tomsick, Laura Sauerbeck, et al. Early hemorrhage growth in patient with intracerebral hemorrhage. Stroke. 1997;28(1):1-5. doi: 10.1161/01. STR.28.1.1.

4. Hemphill JC, Bonovich DC, Besmertis L, Geoffrey T, Manley S, Johnston C, et al. The ICH Score: A Simple, Reliable Grading Scale 
for Intracerebral Hemorrhage. Stroke. 2001;32(4):891-897. DOI: 10.1161/01.STR.32.4.891

5. Sorensen AG, Patel S, Harmath C, Bridges S, Synnott J, Sievers A. et al Comparison of Diameter and Perimeter Methods for Tumor Volume Calculation. Journal of Clinical Oncology. 2001;19(2):551-557.

6. Rost NS, Smith EE, Chang Y, Snider RW, Chanderraj R, Schwab K et. al. Prediction of Functional Outcome in Patients with Primary Intracerebral Hemorrhage, the FUNC Score. Stroke. 2008;39(8):23042309. doi: 10.1161/STROKEAHA.107.512202.

7. Weir CJ, Bradford AP, Lees KR. The prognostic value of the components of the Glasgow Coma Scale following acute stroke. QJM. 2003;96(1):67-74. doi.org/10.1093/qjmed/hcg008.

8. Tuhrim S, Dambrosia JM, Price TR, Mohr JP, Wolf PA, Hier DB. et al. Intracerebral hemorrhage: external validation and extension of a model for prediction of 30-day survival. Ann Neurol. 1991;29(6):658663. DOI: $10.1002 /$ ana.410290614.

9. Juarez VJ, Lyons M. Interrater reliability of the Glasgow Coma Scale. J Neurosci Nurs. 1995;27(5):283-286.

10. Broderick JP, Brott TG, Duldner JE, Tomsick T, Huster G. Volume of intracerebral hemorrhage: a powerful and easy-to-use predictor of 30-day mortality. Stroke. 1993;24:987-993. doi:10.1161/01. STR.24.7.987.

11. Tuhrim S, Horowitz DR, Sacher M, Godbold JH. Volume of ventricular blood is an important determinant of outcome in supratentorial intracerebral hemorrhage. Crit Care Med. 1992;27(3):617-621. DOI: 10.1097/00003246-199903000-00045.

12. Lampl Y, Gilad R, Eshel Y, Sarova-Pinhas I. Neurological and functional outcome in patients with supratentorial hemorrhages: a prospective study. Stroke. 1995;26:2249-2253. doi:10.1161/01.STR.26.12.2249.

13. Juvela S. Risk factors for impaired outcome after spontaneous intracerebral hemorrhage. Arch Neurol. 1995;52(12):1193-1200. doi:10.1001/archneur.1995.00540360071018.

14. Lisk DR, Pasteur W, Rhoades H, Putnam RD, Grotta JC. Early presentation of hemispheric intracerebral hemorrhage: prediction of outcome and guidelines for treatment allocation. Neurology. 1994;44(1):133-139. doi.org/10.1212/WNL.44.1.133.

15. Lampl Y, Gilad R, Eshel Y, Sarova-Pinhas I. Neurological and functional outcome in patients with supratentorial hemorrhages: a prospective study. Stroke. 1995;26(12):2249-2253. Doi:10.1161/01. STR.26.12.2249. 